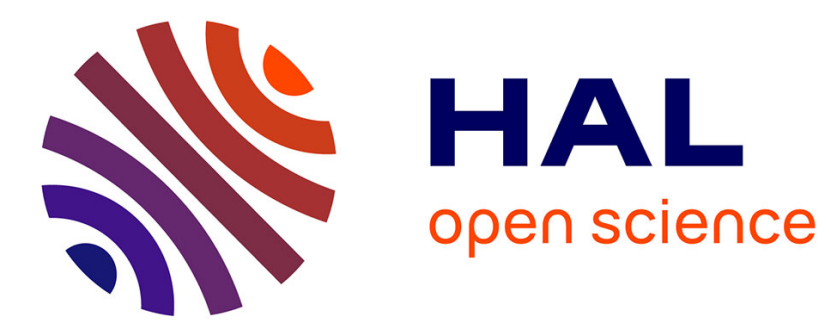

\title{
Détection de larves de poisson anormales par analyse d'images
}

\author{
Sabine Naudin, H. Pella, N. Charlon, Jeanne Garric, Pierre Bergot
}

\section{To cite this version:}

Sabine Naudin, H. Pella, N. Charlon, Jeanne Garric, Pierre Bergot. Détection de larves de poisson anormales par analyse d'images. Aquatic Living Resources, 1996, 9 (2), pp.281-287. hal-02694164

\section{HAL Id: hal-02694164 https://hal.inrae.fr/hal-02694164}

Submitted on 1 Jun 2020

HAL is a multi-disciplinary open access archive for the deposit and dissemination of scientific research documents, whether they are published or not. The documents may come from teaching and research institutions in France or abroad, or from public or private research centers.
L'archive ouverte pluridisciplinaire HAL, est destinée au dépôt et à la diffusion de documents scientifiques de niveau recherche, publiés ou non, émanant des établissements d'enseignement et de recherche français ou étrangers, des laboratoires publics ou privés. 


\title{
Détection de larves de poisson anormales par analyse d'images
}

\author{
Stéphane Naudin ${ }^{(1)}$, Hervé Pella ${ }^{(1)}$, Nicole Charlon ${ }^{(2)}$, \\ Jeanne Garric ${ }^{(1)}$ et Pierre Bergot ${ }^{(2)}$ \\ (1) Laboratoire d'Ecotoxicologie, CEMAGREF, 3 quai Chauveau, 69009 Lyon, France. \\ (2) Adresse actuelle: Springborm Laborratories (Europe) AG, Seestrasse 21, CH-9326 Horn, Suisse. \\ (2) Correspondance: charlon $(9)$ st-pee.inra.fr, \\ Unité mixte INRA-IFREMER de Nutrition des Poissons, \\ Station d'Iydrobiologie, BP 3, 64310 Saint-Pée-sur-Nivelle, France.
}

Reçu le 28 août 1995; accepté le 6 mai 1996.

Naudin S., H. Pella, N. Charlon, J. Garric, P. Bergot. Aquat. Living Resour., 1996, 9, 281-287.

Abnormal fish larvae detection by image analysis.

\begin{abstract}
Image analysis was used for the detection of body deformities in larvae of zebrafish (Brachydanio rerio) and carp (Cyprinus carpio). Live normal and abnormal larvae maintained in water were placed on an illuminated table under a camera and observed in either dorsal or side view. Five morphological descriptors were obtained from two commercial softwares and one specific algorithm. A discriminant analysis was used for the identification of larval position and shape. Preliminary data indicated a good separation between normal larvae in dorsal view and other larvae.
\end{abstract}

Keywords: Image analysis, fish, larvae, Brachydanio rerio, Cyprinus carpio, zebrafish, carp, morphological descriptor, abnormality.

\section{INTRODUCTION}

L'exposition à des substances toxiques ou l'ingestion d'aliments carencés peuvent provoquer chez les larves de poisson des anomalies morphologiques et, en particulier, des déformations de l'axe vertébral ou un gonflement de la cavité péritonéale. Pour les essais de toxicologie comme pour ceux de nutrition, il serait utile de disposer d'une méthode d'identification des larves anormales plus objective et rapide que l'examen visuel. La détection de larves déformées est un problème qui se pose aussi dans les écloseries (Marino et al., 1993; Kiriakos et al., 1995). II semble intéressant de faire appel à l'analyse d'images qui a déjà trouvé de nombreuses applications dans le domaine de l'halieutique, par exemple pour la reconnaissance des espèces de poissons pêchées (Arnarson et al., 1988; Strachan et al., 1990) ou en transit dans des passes à poissons (Castignolles, 1995), le suivi de leur croissance (Poxton et Goldsworthy, 1986; Charlon et al., 1994 ; Tipping, 1994), l'analyse scalimétrique (de Pontual et Prouzet, 1988) ou l'examen de prélèvements planctoniques (Hambright et Fridman, 1994).

L'objectif de ce travail préliminaire est d'examiner la possibilité d'appliquer des techniques simples d'analyse d'images pour distinguer les larves 
déformées des larves normales. Dans une étude précédente, l'identification de larves de carpe présentant des lordoses avait été entreprise sur des animaux anesthésiés, sortis de l'eau et posés sur une surface plane (Geurden et al., 1995). Dans ce travail, les larves ont étć maintenues dans leur milieu, de manière à diminuer le temps de manipulation et l'intensité du stress qu'elles subissent. Plusieurs descripteurs morphométriques, classiques ou issus d'un algorithme de calcul simple, ont été testés. Les essais ont porté sur les larves de deux espèces de cyprinidés souvent utilisées en laboratoire pour des études de toxicologie (danio zébré, Brachydanio rerio) et de nutrition (carpe, Cyprinus carpio).

\section{MATÉRIEL ET MÉTHODES}

\section{Acquisition des images}

Le dispositif d'analyse d'images utilisé est schématisé sur la Figure I. I] comprend une caméra CCD monochrome, un micro-ordinateur (type 486) équipé d'une carte d'acquisition $(512 \times 512$ points images et 256 niveaux de gris entre 0 pour le noir et 255 pour le blanc), un monitcur pour lá visualisation des images et un second moniteur permettant de piloter lc logiciel. Les logicicls utilisés pour l'acquisition d'images et leur traitement sont Visilog 4.1 (Noesis, France) et Optimas 4.1 (Bioscan, USA).

Les animaux examinés sont des larves de danio zébrć âgées de 6 à 8 jours (longueur moyenne 3,7 mm) et des larves de carpe de 20 jours (longueur moyenne $15 \mathrm{~mm}$ ), présentant su non des déformations (Fig. 2).

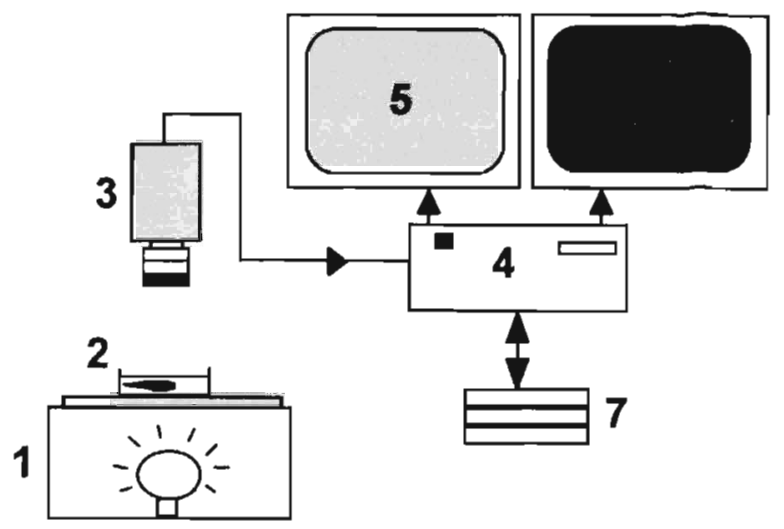

Figure 1, - Installation d'acquisition et de traitement des images (1: table lumincusc ; 2 : échantillon ; 3 : caméra ct objectif ; 4 : ordinateur ; 5: moniteur de visualisation des images; $6:$ monitcur «logiciel»; 7: disques de stockage).

Equipment for image recording and processing (1: illuminated table; 2: sample; 3: camera and lens; 4: computer; 5: video monitor for images; 6: monilor for soffware; 7: storage harddisk).

Les larves sont placées sous la caméra dans un récipient transparent contenant de l'eau (hauteur: $1 \mathrm{~cm})$. La saisie des images des larves est faite par dessus et la définition des contours et le contraste sont optimisés grâce à un rétroéclairage (Fig. 1). Les larves sont vues soit dorsalement lorsqu'elles se tiennent normalement soit latéralement lorsqu'elles sont sur le flanc ou bien couchées sur le fond du récipient.

Pour les images de danio zébré, prises avec Visilog, lc réglage du diaphragme permet de fixer le niveau de gris du fond à environ 230. Le niveau de gris des

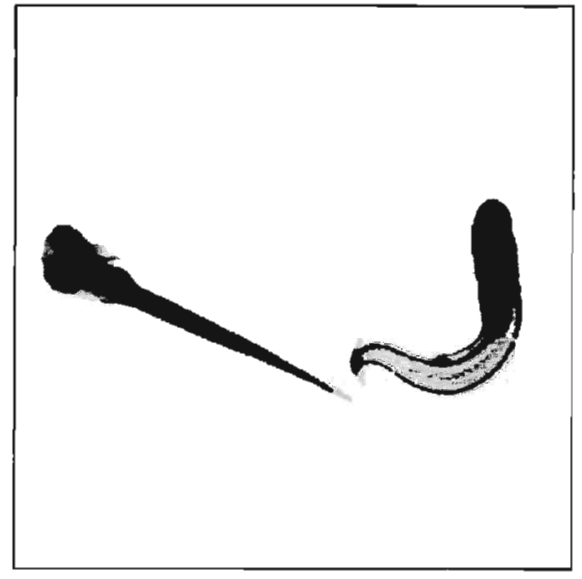

A

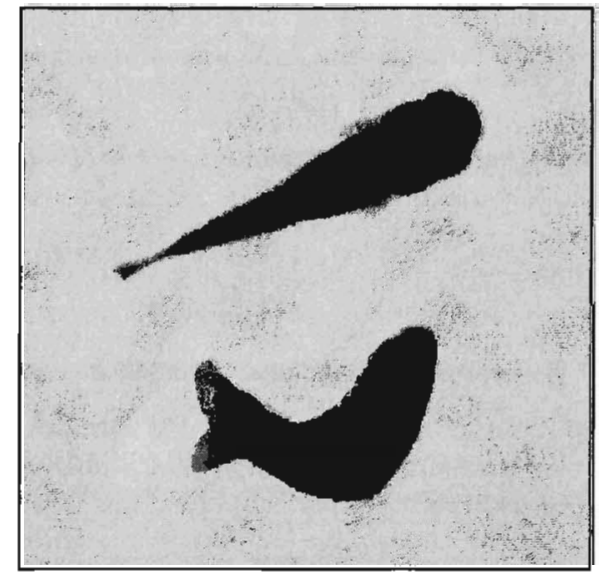

B

Figure 2. - txcmples de larves nomales et anormales de danio zébré (A) et de carpe (B). Image A : à gauche, larve normale en vuc dorsale (longueur $=4,2 \mathrm{~mm}$ ); à àroite, larve déformée en vuc latérale. Image $\mathrm{B}$ : en haut, larve normale en vue dorsale (longueur $=15 \mathrm{~mm}$ ); en bas, larve déformée en vuc latérale.

Examples of nonnal and ahnormal larvae of zebrafish (image A) and carp (image B). Image A: normal larvae on the left (dorsal view, length $=4.2 \mathrm{~mm}$ ), larvae with spinal deformity on the right (side view). Image B: normal larvae on the top (dorsal view, length=15 mm), larvie with spinal deformity on the bollom (side view). 
larves varie alors entre 0 pour les yeux et 210 pour les nageoires. Pour les images de carpe, prises avec Optimas, le niveau de gris du fond est fixé à 150 , les larves ayant un niveau de gris compris entre 0 pour les yeux et 140 pour la partie la plus claire des nageoires.

Les mesures sont réalisées après un étalonnage du système d'acquisition sur les deux axes de l'image pour tenir compte des proportions horizontales et verticales différentes entre le nombre de points images de la caméra: $756 \times 588$ (selon la norme de l'audiovisuel) et celui de la carte d'acquisition: $512 \times 512$. Les mesures corrigées sont ainsi indépendantes de la direction des objets. La reproductibilité des mesures a été vérifiée en prenant dix images d'une même larve avec des directions différentes. Le coefficient de variation trouvé est de $0,5 \%$ pour la surface et de $2,2 \%$ pour le périmètre.

\section{Traitement des images}

Il comprend deux types de traitement.

- La binarisation. Pour éliminer les nageoires tout en conservant les caractéristiques morphométriques du corps, le seuil est fixé à 190 pour le danio zébré et 130 pour la carpe.

- La caractérisation de tous les objets et la sélection des mesures. Un tri est fait sur les critères de surface et de périmètre, ce qui élimine les objets trop petits (particules alimentaires, fèces) ou trop gros (poissons en contact) et conserve les larves isolées. Pour les deux espèces de poissons, la surface, le périmètre et les coordonnées des points du contour sont extraits avec Visilog. La longueur et la largeur du rectangle minimum d'encadrement (RME) qui entoure l'objet parallèlement à ses axes principaux sont extraits avec Optimas.

Ces mesures permettent le calcul des trois descripteurs morphométriques suivants:

- facteur de forme (ou circularité) $=$ périmètre $^{2} /$ surface, RME,

- rectangularité $=$ surface de l'objet/surface du

- élongation = longueur du RME/largeur du RME.

Deux autres descripteurs de déformation sont calculés grâce à un algorithme spécifique:

- rapport entre la taille du squelette et la taille minimum,

- position de la cassure.

L'algorithme utilise le fichier des points du contour des larves, fourni par Visilog (Fig. 3). Le centre de gravité (CDG) est d'abord calculé. L'extrémité de la queue $(Q)$ est déterminée en recherchant le point du contour le plus éloigné du CDG. L'extrémité de la tête $(\mathrm{T})$ correspond au point le plus éloigné du point Q. La taille « minimum » $(\mathrm{Tm})$ de la larve est définie comme étant la distance entre les points $\mathrm{T}$ et $\mathrm{Q}$.

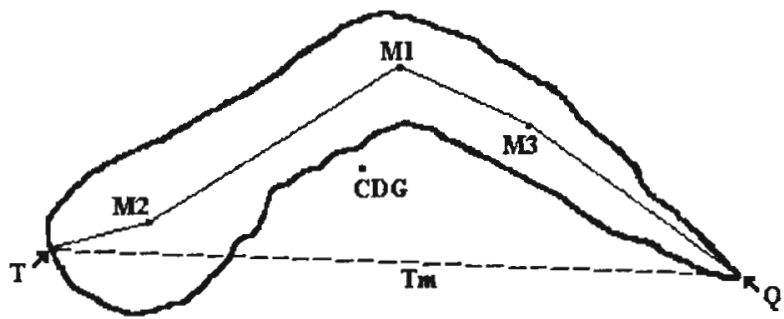

Figure 3. - Hlustration des descripteurs ottenus par l'algorithme spécitique.

Ilustration of the descriptors obtained by the specific algorithm.

Il est alors procédé à un balayage du contour selon une droite perpendiculaire à la droite TQ. A chaque couple de points du contour rencontré, est associć un point moyen, à égale distance des deux points du contour. Le point moyen le plus éloigné de la droite TQ est ensuite déterminé (M1), puis les points moyens intermédiaires $\mathrm{M} 2$ et $\mathrm{M} 3$, respectivement les plus éloignés des droites TM1 et M1Q. Le squelette de chaque larve est défini par quatre segments de droite TM2, M2M1, M1M3 et M3Q et sa taille (Ts) par la somme des longucurs de ces segments. Le premier descripteur de déformation est le rapport des deux tailles calculées Ts/Tm, qui est d'autant plus grand que la larve est plus déformée. Le second descripteur est basé sur le niveau de cassure et correspond au rapport des longueurs $[T M I] /[(T M 1)+(\mathrm{M} 1 \mathrm{Q})]$ exprimé en pourcentage.

\section{Analyse des données}

Les analyses portent sur 66 larves de danio zébré et 38 larves de carpe. Pour chaque espèce, quatre groupes (ND, NL, AD, AL) sont considérés, suivant la forme normale (N) ou anormale (A) des larves, observées en vue dorsale (D) ou latérale (L). Les larves de forme normale en vue latérale correspondent à des individus couchés sur le flanc, qui peuvent être observés lors de tests de toxicologie ou dans certains cas de carences nutritionnelles. Les valeurs moyennes des cinq descripteurs sont comparées dans les différents groupes à l'aide des logiciels Statitcf (ITCF, 1988) ou Toxstat 3.0 (Gulley et al., 1989). Pour mettre en évidence les descripteurs prépondérants, chez les deux espèces, et leur redondance éventuelle, une analyse en composantes principales portant sur l'ensemble des 104 larves est effectuée grâce au logiciel ADE, en utilisant les données centrées réduites (Chessel et Doledec, 1992).

La capacité des descripteurs à distinguer les différents groupes de larves (ND, NL, AD, AL) est testée en scindant les échantillons de chaque espèce en un échantillon d'apprentissage, extrait de façon aléatoire, (comprenant arbitrairement 37 larves pour le danio zébré et 22 larves pour la carpe) et un échantillon d'essai constitué par les larves restantes 
Tableau 1. - Valcurs moyennes des descripteurs pour les différents groupes analysés; ND, NI: formes normales en vue dorsale ou latérale: AD, AL: formes anormales en vue dorsale uu latérale (écart-lype des ćchantillons entre parenthèses) et valcur du test $F$ de l'analyse de variance à un factcur (probabilité entre parenthèses).

Mean values of descriptors for the different grups; ND. NL: normal forms in dorsal or lateral view: AD, AL: abnormal forms in dorsal or lateral view (in brackets: sample standard deviation) and $F$ values of the one way ANOVA (in brackets: probability).

\begin{tabular}{|c|c|c|c|c|c|}
\hline & & & & & \\
\hline Effectif & 21 & 7 & 17 & 21 & $F_{3.62}$ \\
\hline Jacteur de forme (f_f) & $61,1(3,5)$ & $46,2(2,8)$ & $44,4(9,0)$ & $31,7(5,9)$ & $81(<0,001)$ \\
\hline Rectangularité (rec) & $0,48(0,02)$ & $0,58(0,04)$ & $0,43(0,08)$ & $0,51(0,11)$ & $7(<0,001)$ \\
\hline Nivcau de cassure (cass) & $37(26)$ & $50(15)$ & $57(15)$ & $47(16)$ & $3(0,026)$ \\
\hline Cyprinus carpio & & & & & \\
\hline IEffectif & 19 & 4 & 6 & 9 & $F_{3.34}$ \\
\hline Facteur de forme ( $f$ ff) & $51.7(6,7)$ & $34,7(2,8)$ & $47,6(5.7)$ & $39,7(7,3)$ & $11(0,001)$ \\
\hline Rectangularité (rec) & $0.52(0,04)$ & $0,59(0,04)$ & $0,49(0,05)$ & $0.46(0.05)$ & $8(0,001)$ \\
\hline
\end{tabular}

(29 pour le danio zébré et 16 pour la carpe). Une analyse factoriclle discriminante correspondant à une combinaison linéaire des variables (logiciel Statitcf) est pratiquée sur l'échantillon d'apprentissage. Cette analyse fournit, dans l'espace défini par les trois axes discriminants orthogonaux, les coordonnées du centre de gravité de chacun des quatres groupes, les coordonnées de chaque individu et la distance de chaque individu à chacun des quatre centres. Chaque larve, de l'échantillon d'apprentissage ou de I'échantillon d'essai, est classée dans le groupe (ND, NL, AD ou AL) dont le centre de gravité est le plus proche (groupe d'affectation résultant du classement). Une bonne correspondance entre le groupe d'origine (groupe d'appartenance connu avant l'analyse) et le groupe d'affectation indique un tri efficace.

\section{RÉSULTATS}

Le Tableau 1 indique les valeurs moyennes des descripteurs trouvées pour les différents groupes de larves des deux espèces et la signification du test $F$ de comparaison de ces moyennes données par une analyse de variance à un facteur. Des différences très significatives $(p<0,01)$ entre les moyennes des quatre groupes sont trouvées pour les quatre premiers descripteurs chez les deux espèces. Le descripteur "niveau de cassure " montre une différence entre les moyennes faiblement significative $(p=0,026)$ chez le danio zébré et non significative $(p=0,268)$ chez la carpe. La transformation angulaire $\left(y=\arcsin x^{0.5}\right)$ de ce dernier descripteur modifie peu les résultats ( $p=0,037$ et $p=0,201$, respectivement). Les valeurs moyennes des descripteurs «facteur de forme» et
« rectangularité » paraissent influencées à la fois par l'état (normal ou anormal) et la position des larves. Le descripteur "élongation " présente chez le danio zébré des valeurs moyennes beaucoup plus élevées chez les larves normales (ND et NL) que chez les larves anormales (AN et $\mathrm{AL}$ ), indépendamment de leur position. Chez la carpe, ce descripteur distingue le groupe des larves normales en vue dorsale (ND) des autres groupes et notamment des larves normales en vue latéralc (NL). Chez les deux espèces, la valeur moyenne du descripteur "rapport de taille" est la plus faible dans le groupe ND.

L'analyse en composantes principales (ACP) prenant en compte les valeurs des cinq descripteurs

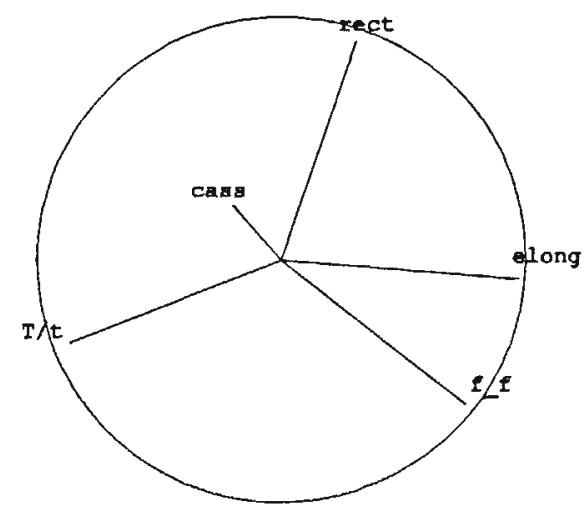

Figure 4. - Cercle de corrélation pour les axes F1 et F2 de l'analyse en composantes principales appliquée aux cinq descripteurs. Correlation circle on the $F I$ and $F 2$ axes from the principal component analysis for the five descriptors. 
et portant sur l'ensemble des larves des deux espèces indique que les deux premiers axes principaux (F1 et F2) expliquent environ $74 \%$ (respectivement 48 et $26 \%$ ) de la variabilité du nuage de points. Le cercle des corrélations pour les axes F1 et F2 (Fig. 4) montre une corrélation importante des variables « élongation (elong) » et «facteur de forme (f_f $)$ avec l'axe F1 ( $r=0,97$ et 0,73 respectivement). La variable " rapport de taille $(\mathrm{T} / \mathrm{t})$ » présente aussi une corrélation importante et négative $(r=-0,87)$ avec cet axe. L'axe F2 est corrélé essentiellement avec la variable « rectangularité (rect) » $(r=0,88)$. Ces quatre variables s'avèrent importantes pour caractériser la forme des larves.

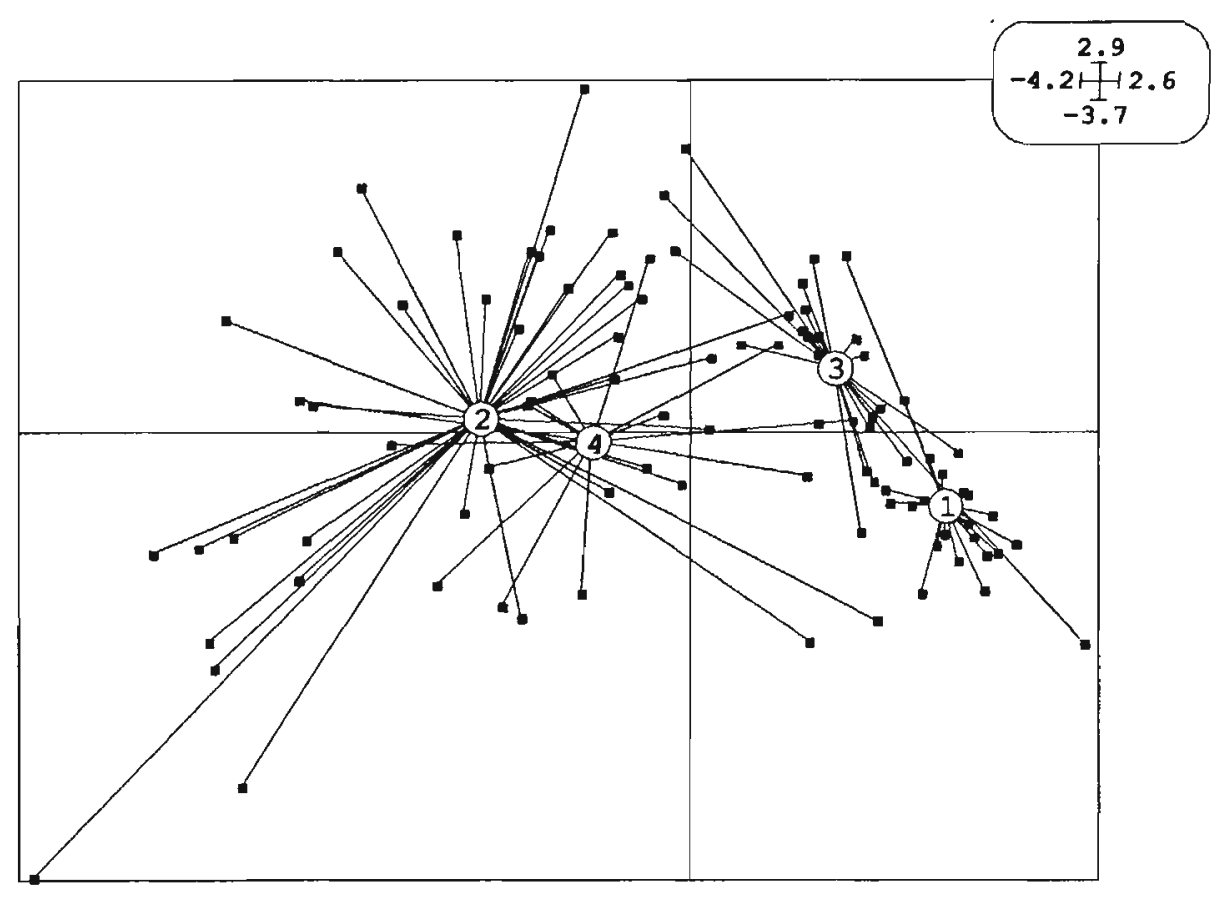

Figure 5. - Représentation des coordonnées factorielles des individus (carrés pleins) dans le plan (F1, F2). Les larves d'un même groupe sont reliées à leur centre de gravité (cercles numérotés) $1:$ danios zébrés normaux, $2:$ danios zébrés anormaux, $3:$ carpes normales, $4:$ carpes anormales. Individuals (filled squares) are positioned on the F1 and F2 plane according to their factorial coordinates. Each circle represents the weighted average of each lavae group coded in the following order: 1: normal zebrafish, 2: abnormal zebrafish, 3: normal carp, 4: abnormal carp.

Tableau 2. - Tableau d'appartenance (lignes: groupes d'appartenance ; colonnes: groupes d'affectation) et nombre d'individus bien classés par l'analyse discriminante, rapporté à l'effectif du groupe d'appartenance (entre parenthèses : probabilité en \%).

Property table (lines: property groups; raws: allocation groups) and number of individuals well classified by discriminant analysis, relative to the number in the property group (in brackets: probability in percentage).

\begin{tabular}{|c|c|c|c|c|c|c|c|c|c|c|c|c|}
\hline & \multicolumn{4}{|c|}{ Echantillon d'apprentissage } & \multicolumn{2}{|c|}{ Bien classés } & \multicolumn{4}{|c|}{ Echantillon d'essai } & \multicolumn{2}{|c|}{ Bien classés } \\
\hline & ND & NL & $\mathrm{AD}$ & $\mathrm{AL}$ & & & ND & NL & $\mathrm{AD}$ & $\mathrm{AL}$ & & \\
\hline \multicolumn{13}{|c|}{ Brachydanio rerio } \\
\hline ND & 10 & 0 & 0 & 0 & $10 / 10$ & $(100)$ & 11 & 0 & 0 & 0 & $11 / 11$ & $(100)$ \\
\hline NL & 0 & 7 & 0 & 0 & $7 / 7$ & $(100)$ & - & - & - & - & - & - \\
\hline $\mathrm{AD}$ & 1 & 0 & 7 & 2 & $7 / 10$ & (70) & 0 & 0 & 5 & 2 & $5 / 7$ & (71) \\
\hline $\mathrm{AL}$ & 0 & 2 & 1 & 7 & $7 / 10$ & $(70)$ & 0 & 0 & 3 & 8 & $8 / 11$ & (73) \\
\hline Total & & & & & $31 / 37$ & $(84)$ & & & & & $24 / 29$ & (83) \\
\hline \multicolumn{13}{|c|}{ Cyprinus carpio } \\
\hline ND & 6 & 0 & 0 & 0 & $6 / 6$ & $(100)$ & 13 & 0 & 0 & 0 & $13 / 13$ & $(100)$ \\
\hline NL & 0 & 4 & 0 & 0 & $4 / 4$ & (100) & - & - & - & - & - & - \\
\hline $\mathrm{AD}$ & 1 & 1 & 3 & 1 & $3 / 6$ & (50) & - & - & - & - & - & - \\
\hline $\mathrm{AL}$ & 0 & 1 & 0 & 5 & $5 / 6$ & (83) & 0 & 0 & 2 & 1 & $1 / 3$ & (33) \\
\hline Total & & & & & $18 / 22$ & $(82)$ & & & & & $14 / 16$ & $(88)$ \\
\hline
\end{tabular}


Les coefficients de corrélation de la variable « niveau de cassure (cass) » avec les axes F1 et F2 sont faibles $(r=-0,19$ et 0,28 respectivement). Les résultats de l'ACP, comme les résultats du Tableau 1 indiquent que les quatre premiers descripteurs sont importants pour caractériser la forme des larves et que le cinquième l'est moins.

La Figure 5 montre les coordonnées factorielles des individus appartenant aux quatre groupes constitués par les larves normales et anormales des deux espèces, sans faire de distinction cntre vue dorsale et vue latérale. Cette figure illustre la dispersion plus importante des individus anormaux, le chevauchement des nuages ainsi que la répartition relative des groupes dans le plan (F1, F2).

Concernant l'analyse discriminante, les résultats du classement des individus des échantillons d'apprentissage et des échantillons d'essai, après affectation au groupe (ND, NL, AD ou $A L$ ) dont le centre de gravité est le plus proche dans l'espace défini par les axes discriminants de l'échantillon d'apprentissage, sont présentés dans le Tableau 2.

Malgré le petit nombre d'individus utilisés dans chaque espèce, l'analyse discriminante sépare correctement une proportion importante des larves des échantillons d'apprentissage, le classement étant cependant meilleur pour les larves normales (groupes ND et NL) que pour les larves anormales. Dans les 'échantillons d'essai, qui n'interviennent pas dans le calcul des axes discriminants, le classement est correct pour les individus du groupe ND (11/11 pour le danio zébré et 13/13 pour la carpe ) mais moins satisfaisant pour les individus des groupes $\mathrm{AD}$ et $\mathrm{AL}$. Ces derniers sont cependant distingués sans erreur des individus du groupe ND.

\section{DISCUSSION ET CONCLUSION}

Les résultats préliminaires obtenus montrent que des techniques simples d'analyse d'images peuvent être utilisées pour distinguer de façon objective les larves normales en vue dorsale de celles qui présentent des anomalies de forme ou de celles qui sont couchées sur le flanc.

Les conditions d'éclairage utilisées donnent un contraste important entre le fond et le corps des larves, au moins pour les deux espèces examinées et permettent de réaliser des séries d'images dans des conditions standardisées. L'étape du choix du scuil reste cependant un obstacle à l'automatisation du traitement des images. Le seuil de binarisation est en effet fixé par l'expérimentateur et doit être adapté à l'espèce et au stade de développement de façon à faire disparaître complètement les nageoires sans élimincr la partie caudale la plus mince du corps des larves.

Dans ce travail, les larves utilisées avaient le même âge et le même stade de développement au sein de chaque espèce et les descripteurs ont été considérés comme étant indépendants de la taille du poisson. Comme la conformation des larves change au cours du développement et évolue progressivement d'un aspect allongé à une forme trapue (Osse et Van den Boogaart, 1995), l'analyse de lots hétérogènes nécessite l'étude préalable de l'influence de la taille du poisson sur les descripteurs. Ainsi dans une étude sur des larves de carpes plus âgécs et plus hétérogènes que dans ce travail, la largeur du RME était proportionnelle à la surface élevée à la puissance 0,52 (Geurden et al., 1995) et non pas 0,50 comme il est attendu en cas de croissance sans changement de forme.

La méthode de tri par affectation des individus à des groupes pré-définis, suivant la distance entre les individus et le centre de gravité de ces groupes, est une méthode classique de décision (Guizard et al., 1992). Dans le cas de la détection des déformations chez les larves, elle suppose de disposer de lots de référence constitués par des animaux de la même espèce et du même stade moyen que ceux de la série à trier, ces lots étant simulés dans ce travail par les groupes d'apprentissage pris dans l'échantillon disponible. Des échantillons plus importants sont nécessaires pour pouvoir évaluer précisément les performances de la méthode. Pour la suite du travail, il paraît aussi utile de définir des groupes d'apprentissage distincts pour les larves présentant un gonflement et pour celles présentant une déformation de la colonne vertébrale, ces deux types d'anomalies pouvant se traduire par des valeurs différentes des descripteurs. Sur les cing descripteurs pris en compte, seul le niveau de cassure s'est avéré peu discriminant. Des descripteurs nouveaux peuvent être envisagés, en tenant compte par exemple des angles formés par le squelette calculé par l'algorithme utilisé dans cette étude.

\section{Remerciements}

Les autcurs remercient tout particulièrement Hervé Capra pour son appui au niveau de l'analyse en composantes principales. 


\section{RÉFÉRENCES}

Arnarson H., K. Bengoetxea, L. F. Pau 1988. Vision applications in the fishing and fish product industries. Intl. J. Pattern Recognit. Artif. Intell. 2, 657-671.

Castignolles N. 1995. Automatisation du comptage et de la reconnaissance des espèces dans les passes à poisson par analyse de sćquences d'images. Thèse dr. $3 \mathrm{c}$ cycle, INP Toulouse, $172 \mathrm{p}$.

Charlon N., J.-C. Folmer, P. Laborde 1994. Application de l'analyse d'image à l'étude de la croissance de larves de poisson. In: L'application de l'analyse d'images dans l'agro-industrie. C. Guizard, D. Bertrand eds. CEMAGREF, 17-23.

Chessel D., S. Doledec 1992. Software multivariate analyses and graphical display for environmental data (version 3.4). Université Lyon I, 523 p.

de Pontual H., P. Prouzet 1988. Numerical analysis of scale morphology to discriminate between Atlantic salmon stocks. Aquat. Living Resour. 1, 17-27.

Geurden I., N. Charlon, D. Marion, P. Bergot 1995. Dietary phospholipids and body deformilies in carp (Cyprinus carpio L.) larvae. Larvi'95, Fish and crustacean larviculture. Symp. Europ. Aquac. Soc. Spec. Publ. 24, 162-165.

Guizard C., V. Bellon, F. Sevila 1992. Vision artificielle dans les industries agro-alimentaires. Editions CEMAGREFDicova, Antony, 279 p.

Gulley D. D., A. M. Boelter, H. L. Bergman 1989. Toxstat Release 3.0. Fish physiology and toxicology laboratory,
Department of zoology and physiology. Univ. Wyoming, U.S.A.

Hambright K. D., S. Fridman 1994. A computer-assisted plankton analysis system for the Macintosh. Fisheries 19, 6-8.

ITCF 1988. Statitcf. 4ème éd. Scrvices des Etudes statistiques, Paris.

Kiriakos Z., G. Koumoundouros, P. Divanach, M. Kentouri 1994. Prelarval and larval description of notochord development as a criterion for early detection of tail abnormalities in gilthead seabream (Sparus aurata). In: Measures for success. P. Kestemont, J. Muir, F. Sevila, P. Williot cds. CEMAGREF, 117-118.

Marino G., C. Boglione, B. Bertolini, A. Rossi, F. Ferreri, S. Cataudella 1993. Observations on development and anomalies in the appendicular skeleton of sea bass, Dicentrarchus labrax L., 1758, larvae and juveniles. Aquac. Fish. Manage. 24, 445-456.

Osse J. W. M., J. G. M. Van den Boogaart 1995. Fish larvae, development, allometric growth, and the aquatic environment. ICES Mar. Sci. Symp. 201, 21-34.

Poxton M. G., G. T. Goldsworthy 1986. The remote estimation of weight and growth in turbot using image analysis. In : Automation, data processing in aquaculture. J. G. Balchen ed., 163-170.

Strachan N. J. C., P. Nesvadba, A. R. Allen 1990. Fish species recognition by shape analysis of images. Pattern Recognit. 23, 539-544.

Tjpping J. M. 1994. Measuring fish by video image processing. Progress. Fish-Cult. 56, 299-300. 